\title{
Evolution and Reengineering of NASA's Flight Dynamics Facility (FDF)
}

\author{
Thomas Stengle ${ }^{1}$ and Susan Hoge. ${ }^{2}$ \\ NASA Goddard Space Flight Center, Greenbelt, MD 20771USA
}

\begin{abstract}
The NASA Goddard Space Flight Center's Flight Dynamics Facility (FDF) is a multimission support facility that performs ground navigation and spacecraft trajectory design services for a wide range of scientific satellites. The FDF also supports the NASA Space Network by providing orbit determination and tracking data evaluation services for the Tracking Data Relay Satellite System (TDRSS). The FDF traces its history to early NASA missions in the 1960's, including navigation support to the Apollo lunar missions. Over its 40 year history, the FDF has undergone many changes in its architecture, services offered, missions supported, management approach, and business operation. As a fully reimbursable facility (users now pay $100 \%$ of all costs for FDF operations and sustaining engineering activities), the FDF has faced significant challenges in recent years in providing mission critical products and services at minimal cost while defining and implementing upgrades necessary to meet future mission demands. This paper traces the history of the FDF and discusses significant events in the past that impacted the FDF infrastructure and/or business model, and the events today that are shaping the plans for the FDF in the next decade. Today's drivers for change include new mission requirements, the availability of new technology for spacecraft navigation, and continued pressures for cost reduction from FDF users. Recently, the FDF completed an architecture study based on these drivers that defines significant changes planned for the facility. This paper discusses the results of this study and a proposed implementation plan. As a case study in how flight dynamics operations have evolved and will continue to evolve, this paper focuses on two periods of time (1992 and the present) in order to contrast the dramatic changes that have taken place in the FDF. This paper offers observations and plans for the evolution of the FDF over the next ten years. Finally, this paper defines the mission model of the future for the FDF based on NASA's current mission list and planning for the Constellation Program. As part of this discussion the following are addressed: the relevance and benefits of a multi-mission facility for NASA's navigation operations in the future; anticipated technologies affecting ground orbit determination; continued incorporation of Commercial Off-the-Shelf (COTS) software into the FDF; challenges of a business model that relies entirely on user fees to fund facility upgrades; anticipated changes in flight dynamics services required; and considerations for defining architecture upgrades given a set of cost drivers.
\end{abstract}

\section{Introduction}

The Flight Dynamics Facility (FDF) located at the Goddard Space Flight Center (GSFC) in Greenbelt, Maryland is an essential NASA institutional resource that provides multi-mission navigation services for a wide range of robotic and human space flight missions. Navigation services include orbit determination, maneuver planning and orbit product generation, including the computation and delivery of acquisition data (station pointing data). The FDF has a long history of flight project support and has long term commitments to future NASA projects and programs.

Today, the FDF provides routine ground based orbit determination for eighteen satellites. Included in this mission set are science spacecraft such as the Hubble Space Telescope (HST) and the Solar TErrestrial RElations Observatory (STEREO). Orbit determination is also provided for the NASA Tracking and Data Relay Satellite System (TDRSS). The FDF routinely supports missions in flight regimes that include Low Earth, Geosynchronous, Lunar, Libration Point and Heliocentric orbits. The FDF will add mission support for the Solar Dynamics Observatory (SDO), Lunar Reconnaissance Orbiter (LRO) and Gamma-ray Large Area Space Telescope (GLAST)

\footnotetext{
${ }^{1}$ Head, Flight Dynamics Analysis Branch, Code 595

${ }^{2}$ Flight Dynamics Facility Operations Director, Code 595
} 
missions that are scheduled for launch in 2008. In addition to science mission support, the FDF is an important component of the ground support infrastructure for NASA's human space flight program. The FDF provides routine orbit determination for the International Space Station (ISS), serves as the backup facility for Space Shuttle (STS) orbit determination, and generates acquisition data for these two programs. The FDF also provides navigation products and services to other government agencies such as the National Oceanic and Atmospheric Administration (NOAA), and supports NASA missions operated at universities and other non-NASA sites such as the University of California at Berkeley and the Applied Physics Laboratory.

Future NASA science missions will continue to use the FDF for navigation products and services. This includes missions such as the Magnetospheric Multiscale Mission (MMS)

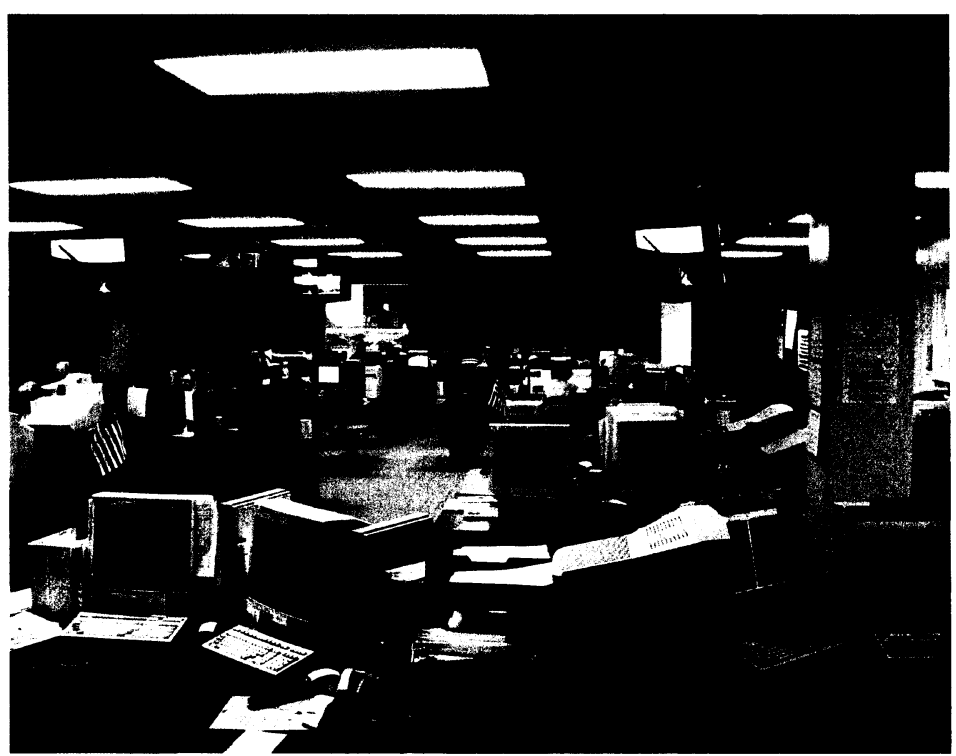

Figure 1. FDF Operations Room and the James Webb Space Telescope (JWST). In order to be responsive to future navigation requirements, the FDF must continue to evolve in order to leverage new technology and present FDF users with cost effective solutions for ground-based navigation support.

The FDF is a fully self-sustaining operation in which users pay for all services and fund all sustaining engineering and facility operations activities. In addition to the future technical challenges that face the operation of the FDF, the present business operation of the FDF presents management challenges as well, including the planning of major upgrades to the facility. Today, all incremental or major upgrades to the facility must be funded by existing and/or future FDF users.

The purpose of this paper is to examine the past evolution of the FDF for lessons learned and to present current plans for the "fourth generation" FDF in response to recent and anticipated changes in the future. Fundamental to this discussion is whether a multi-mission facility for ground-based navigation computations still serves a purpose that can justify the significant changes that are planned to reengineer the current FDF architecture.

\section{The FDF: Current Products and Services}

The FDF provides orbit determination; acquisition data generation and transmission; planning product generation; tracking data evaluation and certification; and maneuver planning and calibration services on a regular basis. "Full-service" orbit determination is provided for 18 missions. These are typically missions that operate and require FDF products and services for many years over the lifetime of the spacecraft. Station pointing data (acquisition data) is provided on a regular basis for about 25 missions. This acquisition data is sent to the Space Network (SN), Ground Network (GN), Deep Space Network (DSN) and the Universal Space Network (USN). Planning products such as station view periods and spacecraft eclipse predictions are provided to various Mission Operations Centers (MOCs) to be used for station scheduling and operations planning. Tracking data evaluation is performed on a regular basis for the $\mathrm{SN}$ and the $\mathrm{GN}$ and as part of the launch and early orbit support provided by the FDF. Tracking data validation support is also provided for new tracking sites or those that have upgraded their equipment. Most recently this service was provided for the LRO and SDO projects. Maneuver planning and calibration support are provided for several legacy missions, however for most new missions this function is performed in the MOC.

In addition to full-service missions, the FDF provides support to missions on a short-term or special support basis. This support usually covers the launch and early orbit period or times when a spacecraft experiences an anomaly. An example of this type of support is a near-Earth spacecraft that uses the Global Positioning System (GPS) for navigation. The FDF can provide navigation and acquisition data services to missions for the first 3-5 days after launch until the onboard GPS solutions are verified. The FDF provides support for approximately 12 expendable launch vehicles (ELVs) in a calendar year. Support provided includes pre-launch generation of acquisition data; near-real-time powered flight data monitoring; updated acquisition data generation and 
transmission; and separation vector generation and transmission. The FDF regularly supports Sealaunch, Atlas V, and Delta IV launch vehicles carrying both NASA and commercial payloads.

The FDF has a support role for STS and ISS. The STS support consists of providing acquisition data to SN and GN and other sites that support STS. This data includes both nominal acquisition data and contingency acquisition data. ISS support consists of processing tracking data and providing orbit vectors to NASA's Johnson Space Center (JSC) for the ISS and acquisition data to the networks. The FDF also provides tracking data evaluation to ISS and STS. In addition, the FDF is back-up to JSC for STS navigation for emergency situations.

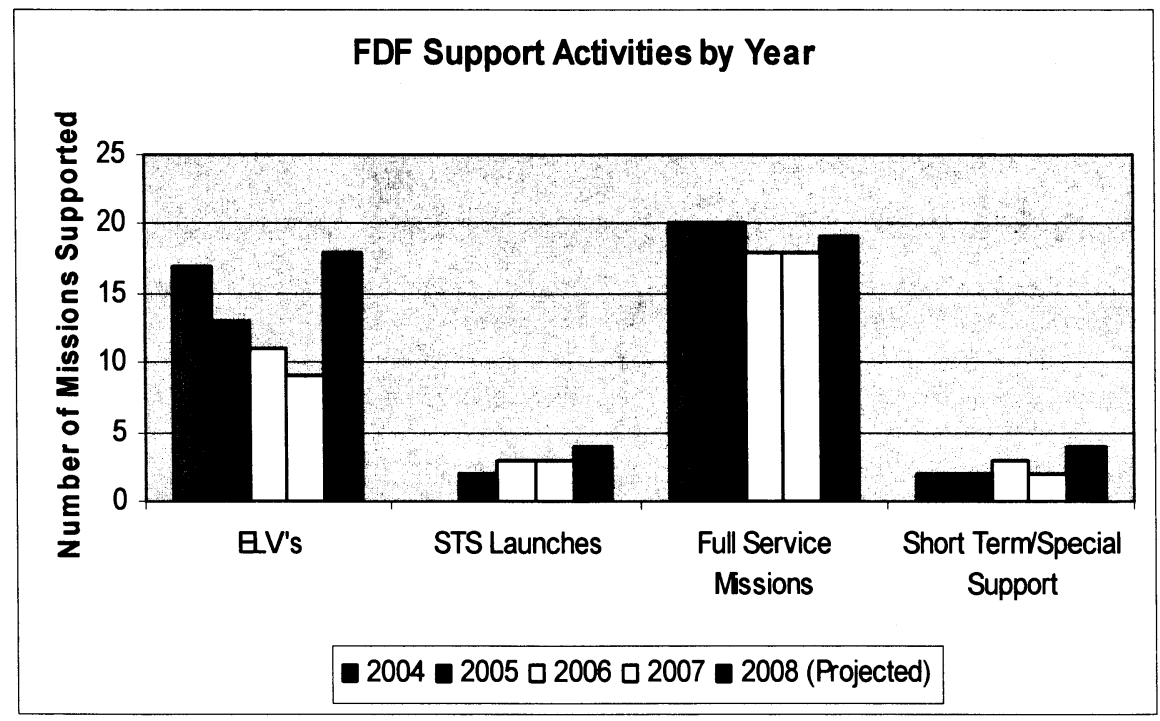

Figure 2. Present FDF Operations Load

The mission support products generated by the FDF are delivered to users via the FDF product server. Orbit planning information is placed on the server so that missions may pull their products when needed. Products delivered through the product server include mission planning products, station view periods, state vectors and ephemerides. Some acquisition data is delivered through the product server, however, most acquisition data is sent directly to the required network.

The FDF has moved toward generation of a standard set of products over the last several years and away from supplying project-unique products. With the availability of commercial software that produces many of the products that the FDF traditionally provided using in-house software, it is easier and more cost-effective for the missions to receive a standard set of products or ephemeris from the FDF and then generate their own unique set of products. This allows the user to customize the product set to their needs and to generate products when needed. It also minimizes the number of special requests that the FDF needs to provide.

The support outlined above is provided using various computing platforms within the FDF. The FDF has approximately 70 machines that are a mixture of UNIX and Windows-based servers and workstations. These computers provide the application computing, communications, and data processing support for the facility. The FDF has a distributed computing architecture with about one third of the machines supporting infrastructure services such as communications, data processing and storage and system monitoring. The remaining machines are used for operations. When critical operations are performed, processing is restricted to certain machines in order to maintain strict configuration control.

The FDF interfaces with the GSFC Internet Protocol Operational Network (IONet) for its operations. The IONet is divided into three subnets. The most secure is the Closed IONet where the FDF performs analysis and operations. The Restricted IONet and the less-restricted Open IONet make up the other two segments of the IONet. These network connections allow the FDF to reach its customers and provide for data exchange, delivery of products or the receipt of data. The FDF interfaces with a wide range of external entities through these networks, including White Sands Complex (WSC) for SN support; NASA, U.S. Department of Defense and commercial ground networks; launch support facilities; and Mission Control Centers. Data formats and delivery methods vary depending on the external interface and entity. 


\section{Evolution of the Current FDF}

The FDF has undergone tremendous change over its long history. In the last 15 years alone, the facility has evolved from a mainframe computer facility with institutional funding to a distributed computing environment facility that is completely user funded. This change has occurred gradually, and for the most part unintentionally and without a systematic process or plan. As a result, the change has left the facility with a system that is often difficult to change quickly to meet future mission needs.

\section{A. The Early Years of the FDF}

The origins of the FDF trace back to the earliest days of the United States space program. In the 1960's, groundbased orbit determination at GSFC was performed by two separate organizations in order to satisfy two distinctly different requirements. The first group provided orbit determination for GSFC's early science missions to enable science data processing, spacecraft maneuver planning and scheduling of network resources. The second group was part of the original manned space flight ground network. In support of the Apollo program, this group's role was to process tracking data for purposes of validating the data quality of the Apollo Manned Space Flight Network (MSFN), providing acquisition data for that network and evaluating the quality of this data for navigation computations at JSC. A byproduct of this process was an orbit determination product that could be used as a backup to the product generated by the JSC navigation team. This dual role of providing navigation services to science missions as well as supporting the NASA Ground and Space Networks with tracking data evaluation services continues today.

By the mid-1970's the two navigation groups at Goddard coalesced into a single group and through various organization changes and consolidation of operations facilities, the entity that we now identify as the FDF came into existence in 1985. The facility that existed in the 1980's consisted of IBM compatible mainframe computers and applications software developed at Goddard. Most of this software was FORTRAN based.

\section{B. FDF Upgrade Planning in the Early 1990's}

In 1992 the FDF was a modern operations facility that supported a large number of flight projects and the tracking networks. Critical operations, routine operations, and contingency operations were supported out of two large operations rooms. The architecture was mature and featured three mainframe computers used for all operational support as well as analysis and development activities. Each mainframe was rated at 8.4 million instructions per second (MIPS) and had 32 megabytes of memory. By today's standards, this compute power seems underwhelming, but it was enough to support a broader range of service requirements than those that exist today.

A "long range" plan ${ }^{1}$ was completed in 1991 to provide a guide "to evolve from current support capabilities to those required to meet identified and projected services for flight projects and the networks." Four goals were identified in the document:

1. Reduce costs

2. Provide FDF users with high accuracy navigation products

3. Maintain a state-of-the-art FDF based on a systematic phased replacement philosophy

4. Develop staff skills

Various near-term ( 5 year) activities were identified to meet these goals. First and foremost was emphasis on reducing overall project support costs by reducing FDF software development and maintenance costs. Software development and maintenance were seen as the major contributor to the overall support costs and an area where savings could be made. This was to be accomplished through an expanded software library, but the primary focus was to be the development of the Combined Operational Mission Planning and Attitude Support System (COMPASS). . While the Goddard Trajectory Determination System (GTDS) used for orbit determination computations was truly generic and supported most new missions with no modification, systems that were required for mission trajectory design and attitude determination often required new development or significant modifications to existing systems. COMPASS was an effort to develop a generalized flight dynamics system for attitude and trajectory design computations that could increase mission-to-mission software reuse to the 80 percent level from the traditional 20 percent level. This generalized software system would result (it was believed) in a 50 percent reduction in the development costs for new and unique navigation, mission design and attitude software systems required for future missions. While this system would effectively replace all of the applications software in the FDF, the projected development cost was enormous (greater than 200 staff-years). This effort was eventually discontinued for this reason as well as the availability of COTS, new software development approaches and changes in spacecraft support requirements that eliminated the need for extensive ground attitude determination computations. Hindsight 
now tells us that there never would have been an appropriate return on investment to justify the enormous cost of this development.

As part of implementing the 1991 long-range plan, a formal FDF architecture study ${ }^{2}$ was completed in 1992 to determine the optimum FDF computing environment based on a projected workload over the next 5 years. A look back at the conclusions of this study is interesting. At the time, the projected workload was expected to increase in complexity and frequency. This 1992 study was grounded in a mainframe perspective and identified an "ideal" architecture that had the following characteristics:

- Portable applications

- Heterogeneous networks of computing platforms (multiple hardware platforms including mainframes) connected together by a Fiber Distributed Data Interface (FDDI) backbone

- Computing platforms that communicate via protocols that comply with Government Open Systems Interconnection Profile (GOSIP) standards.

- Open systems architecture that would be compliant with Portable Operating System Interface for Computer Environments (POSIX)

With respect to the flight dynamics applications that would be executed in the FDF, the 1992 architecture study did not address this in depth but acknowledged the need to continue the COMPASS development effort that was underway. In addition to supporting the COMPASS development, the 1992 architecture study supported continued automation of FDF products, services and operations to the maximum degree practical.

Despite the strong desire by the 1992 architecture study team to move the FDF to the "ideal" architecture, it was felt that this was not a feasible goal to achieve within a 5 year period due to funding constraints and the risk to missions being supported at that time. Another major obstacle noted was the approximately 4 million lines of FDF applications software code that was mainframe and FORTRAN based. This software was designed to execute on an IBM mainframe compatible architecture under the MVSA/XA operating system and would need to be ported to other computing platforms and/or re-architected to take advantage of newer machine architecture and capabilities.

Therefore, the team's final recommendation was to develop a "heterogeneous platform" based architecture that consisted of replacing current mainframes with more powerful mainframes and gradually moving applications software to other platforms in an open systems environment. This was an intermediate step to the "ideal" architecture that might be achieved eventually over a 10 year period. In retrospect, the 1992 architecture study defined an evolutionary path that was reasonable and in line with the direction of computing technology. The study did not, however, address in specific terms the cost of the architecture changes required or present a business case for this change with quantifiable benefits in return for a substantial funding investment. Nor did it develop a realistic mission model for known and potential mission support.

The 1992 architecture study focused on defining an FDF architecture while making a general assumption that the support requirements for missions would remain generally the same. In 1995, an in-house team examined flight dynamics operations concepts of the future and focused on the nature of flight dynamics support in the year 2000 . In its final report ${ }^{4}$ and a subsequent update to the report ${ }^{5}$ in 2003 , key "operations concept elements" were defined that provided guidelines for future operation of the FDF. These had significant implications with regard to the facility and its architecture needs. Included were the following elements:

1. The Mission Operations Centers (MOCs) should be the focal point for all ground attitude determination (real-time and near-real-time), product generation, mission design and maneuver support activities during routine mission operations.

2. The FDF should evolve into an orbit-only operations facility. The FDF should house the core expertise at GSFC in routine ground orbit determination. While orbit determination may be performed in a MOC, this is not the preferred approach. Experience and understanding of orbit determination for various types of orbits could be lost if all MOCs provided their own orbit determination.

3. Strong consideration should be given to onboard orbit determination for future missions. This will require the FDF to serve in a backup capacity. Either the MOC or FDF should perform the quality assurance of the downlinked solution and produce the required mission products as deemed to be most cost-effective.

4. A base set of orbit products that can be provided by the FDF should be established. As part of this effort the number of TDRS products for end users should be standardized.

5. The generation of orbit/attitude event prediction products (e.g. sensor viewing, contact prediction) should be provided by the ultimate user employing COTS (preferred) or in-house supplied and maintained software. 
6. A centralized, multi-mission Flight Dynamics Product Center with automated capability to generate, Quality Assure, and file (accessible by remote users) basic orbit products should continue to evolve and serve as the primary mechanism to allow transfer of FDF orbit determination products to outside entities.

\section{A Look Back: Changes since 1992}

The changes that were implemented as a result of the 1991 long range plan, the 1992 architecture study and the 1995 and 2003 operations concepts paper have brought us to the present FDF. In general, these changes to the FDF kept pace with project support requirements. An examination of the evolution of the FDF during this period presents many lessons for future planning.

Consistent with the operations concept elements defined in the 1995 report, the FDF became more focused as a navigation facility. Attitude determination support within the FDF ended in 2007 due to improvements in onboard capability; changes in mission requirements and operations approach; and new ground processing techniques. For many years, unique ground attitude determination software systems had to be developed and integrated in the FDF for each new mission. This was a significant ground system cost. In the late 1990's, a multi-mission software system was developed in-house using Matlab ${ }^{\circledR}$. This dramatically decreased the mission unique development costs. Its portability and ease of use by MOC Flight Operations Team members resulted in moving the ground attitude determination function to the MOCs. More significantly, onboard systems generally provided attitude knowledge to an accuracy sufficient to decrease dependency on ground based computations.

Out of necessity, the FDF evolved to a distributed computing environment with UNIX and Windows workstations. Various commercial off-the-shelf (COTS) software tools began to displace some of the in-house developed flight dynamics applications software. Between 1995 and 1997, the FDF embarked on an accelerated program to move all applications off of mainframe computers. This was viewed as a step that would result in immediate cost savings by eliminating a sizable staff dedicated to mainframe computer operations and maintenance. While this was consistent with the long term objectives outlined in the 1992 architecture study, this migration did not substantially change the mainframe based architecture and its interfaces and data processing flow. The focus of this effort was to "port" old software applications to workstations. This architecture continues today and can best be described as an aging infrastructure that is a complex mixture of internal and external interfaces that are becoming increasingly expensive to maintain. Its advantage is that it does consist of many proven applications software systems that have successfully supported a large number of past missions (and can continue to support future missions). Its main drawback is its lack of flexibility and cost to maintain and modify. Modifications to data types or interfaces may ripple through many components of the architecture and applications systems.

Another significant change in the 1990's regarded the automation and delivery of FDF orbit products. Significant cost reduction was achieved in the early 1990's through the development and use of the Orbit Production Automation System (OPAS) and Quality Assurance Automation Software (QA Tool). ${ }^{3}$ The late 1990's saw the creation of the FDF Product Center where users could retrieve their products via a web-based interface. Along with this, the number of products produced by the FDF was reduced. The generation of secondary or "ancillary" orbit products that were project unique was generally moved to the MOCs.

While orbit maneuver planning is still performed in the FDF for science missions, the last ten years have seen this function more likely to be hosted in MOCs. This decision is typically based on specific requirements of a flight project and skill needs of the MOC flight operations team. Today, if this function is performed in the MOC, the FDF generally provides backup technical support.

Perhaps the most significant event during the recent history of the FDF that continues to have far reaching and significant impact on the FDF and its future direction involves its business operation. Prior to 1998, the Goddard Flight Dynamics Division (FDD) was responsible for directing all activities associated with the FDF. Although NASA contractor staff provided most of the routine operations support within the FDF, there was active management and direction of this work by the NASA civil servant work force within the FDD. This same civil servant staff provided leadership of FDF modernization efforts as well. During this period, funding for nearly all operations, development and analysis support was provided by NASA Headquarters. Beginning in 1998, NASA began a consolidation of operations contracts, gave the contractor team a greater responsibility in managing operations and removed management of the FDF from the Goddard Flight Dynamics Division. In 2004, the management responsibility for the FDF changed once again. As before, the FDF management became the responsibility of the NASA engineering organization that was home to NASA civil servant flight dynamics engineers (now the Flight Dynamics Analysis Branch). While this presented a number of challenges to the NASA staff, probably the most significant was establishing new business processes to recover operations costs from all FDF users. Funding is no longer provided by NASA Headquarters; the FDF is now required to be a fully selfsustaining operation. 
In 2006, the Flight Dynamics Users Council was established as a key element of the FDF operation. This Council consists of users and sponsors that are beneficiaries of flight dynamics services at GSFC. The primary functions of the Council are to review, critique and reach consensus on support strategies, long range plans and resulting core capabilities and resource needs for operating the FDF. The objective is to enhance the flight dynamics services offered at GSFC and ensure they are responsive to the near term and long term needs of NASA flight projects (robotic and manned), space and ground networks, and technology development programs. Specifically, the Council performs the following functions:

- Serves as advocacy group for long range flight dynamics planning

- Reviews flight dynamics infrastructure requirements and budget requests;

- Reviews/approves FDF reengineering plans

- Represents flight dynamics customers and commits to meeting budget requirements

- Helps assure visibility of FDF costs and budget submittals

- Reviews and provides feedback on FDF business models

Table 1 is a summary and comparison of the FDF between 1992 and the present. Through this period of enormous change, the FDF provided successful support to dozens of missions.

Table 1. Comparison of the FDF: 1992 and the Present

\begin{tabular}{|c|c|c|}
\hline & 1992 & 2008 \\
\hline FDF Services & $\begin{array}{l}\text { Navigation services: orbit } \\
\text { determination, acquisition data } \\
\text { generation, orbit product } \\
\text { generation, orbit maneuver } \\
\text { planning, tracking data } \\
\text { evaluation } \\
\text { Attitude services: attitude } \\
\text { determination, control, } \\
\text { calibration }\end{array}$ & $\begin{array}{l}\text { Navigation services: similar to } \\
1992 \text { services with reduced set } \\
\text { of orbit planning products; } \\
\text { routine orbit maneuver planning } \\
\text { may be performed in MOCs } \\
\text { Attitude services: none } \\
\text { performed within the FDF }\end{array}$ \\
\hline Missions supported & $\begin{array}{l}\text { Full Service: } 12 \\
\text { Short term: } 1 \\
\text { ELVs: records not available } \\
\text { STS: } 8\end{array}$ & $\begin{array}{l}\text { Full Service: } 18 \\
\text { Short term: } 1 \\
\text { ELVs: } 12 \text { planned } \\
\text { STS: } 5 \text { planned } \\
\end{array}$ \\
\hline $\begin{array}{l}\text { Future Mission Model } \\
\text { (new missions projected over } \\
\text { next } 5 \text { years) }\end{array}$ & $\begin{array}{l}10 \text { new full service missions } \\
\text { added between } 1992-1997 ; \text { STS } \\
\text { launches projected for many } \\
\text { years }\end{array}$ & $\begin{array}{l}2 \text { new full service missions } \\
\text { planned between } 2007-2012 ; \\
12 \text { short term missions; STS } \\
\text { support ending with uncertainty } \\
\text { in Constellation Program support }\end{array}$ \\
\hline Approximate Facility Staffing & $\begin{array}{l}175 \\
(50 \% \text { for facility operations, } \\
\text { sustaining engineering, software } \\
\& \text { system maintenance) }\end{array}$ & $\begin{array}{l}55 \\
(50 \% \text { for facility operations, } \\
\text { sustaining engineering, software } \\
\& \text { system maintenance) }\end{array}$ \\
\hline Computer Platforms & Mainframes & Workstations \\
\hline Applications & All In-house & In-house + commercial software \\
\hline Product Delivery & FDF originated transfer & Product Center \\
\hline Ancillary product generation & Primarily in the FDF & Mostly MOC based \\
\hline Funding Source & $\begin{array}{l}\text { Primarily NASA Headquarters } \\
\text { funding }\end{array}$ & $100 \%$ user fees \\
\hline FDF Management & $\begin{array}{l}\text { NASA division level } \\
\text { organization; headquarters } \\
\text { sponsorship }\end{array}$ & $\begin{array}{l}\text { NASA branch level } \\
\text { organization; Flight Dynamics } \\
\text { Users Council for user input }\end{array}$ \\
\hline
\end{tabular}




\section{Anticipating and Responding to Past Change: Lesson's Learned}

In retrospect, the events of the past fifteen years have given us many "lessons learned" that can be applied today as we look to the future of the FDF. Among these are the following:

- "Flight dynamics operations" is an engineering sub-discipline and expertise that must be cultivated and can be easily lost. In-house knowledge of operations interfaces, ground system design and facility architecture is essential for providing effective flight dynamics support to flight projects and leading changes necessary for future mission support.

- A single organization should have end-to-end ownership of all flight dynamics services and operations. This assures the FDF evolves based on a clear understanding of future mission needs, and provides the flight projects with a single point of contact for flight dynamics mission services.

- Realistic analysis of future mission models and operations concepts must drive change for the FDF. The 1992 architecture study had a reasonable view of the direction of facility computer hardware and networks but it failed to take a critical look at both the need and delivery of services in the future. Assessing a realistic mission model for future architecture and support planning presents a challenge and within the FDF there are numerous examples of how we have focused too much or too little on perceived trends in new mission support. As important as it is to predict the types of missions to be supported in the future, it is just as important to not base a major change in direction on a single view of the mission model. An overly optimistic view of the mission set, including increases in numbers of full service missions to support (many formation flying or constellation missions) characterized FDF planning in the late 1990's.

- Facility architecture issues can not be deferred indefinitely. While the accelerated approach to moving off of the mainframe computers achieved a significant level of success with respect to reducing costs of system hardware and maintenance, the "quick and dirty" approach did not address architecture improvements that are now becoming critical for efficient operations in the future.

- COTS software has become an important part of the suite of applications software in the FDF. Much of the focus on cost reduction in the early 1990's was to reduce the cost of in-house software development and maintenance. COTS software, especially for database management, orbit product generation and maneuver planning, has helped accomplish this goal. However, COTS software will not eliminate the need for some in-house developed software.

- Simple and clear strategic plans can provide valuable guidance and direction to an organization. While we often focus on how we fail to anticipate future events, we can point to some correct predictions made (e.g. COTS, changing operations concepts in support of autonomous navigation) that influenced the FDF planning in a positive way.

- A multi-mission operations facility such as the FDF can operate as a fully self-sustaining operation. We believe the services offered are cost-competitive given new customers that have made commitments for future support. While some services provided by the FDF are not critical for the maintenance of core expertise or the development of new and interesting capabilities, they are, nevertheless, important to offer to customers in order to help maintain the FDF infrastructure. Routine activities such as ELV support are important to help maintain a base of funding for the FDF infrastructure. Key to the success of this business approach is active communication with FDF users. The establishment of the Flight Dynamics Users Council was essential for discussing requirements for FDF support and negotiating funding commitments.

- System architectures must be flexible in order to respond rapidly to change. The FDF system architecture envisioned in the early nineties was one that did not change both from a hardware or software perspective. Today, a long hardware lifetime is 10 years, and software needs to be flexible to accommodate multiple operating system changes. Add to that the ever changing IT security needs and any system needs to accept changes without major modifications.

- Spacecraft autonomous navigation systems (e.g. GPS) have begun to change and will continue to change the support requirements for the FDF. This is clearly a positive development for spacecraft navigation and the FDF must continue to adapt to focus on short term (early mission) or anomaly support to Earth orbiting missions with navigation autonomy.

\section{E. The Case For Continuing A Multi-Mission Navigation Facility at Goddard}

Over the last ten years there has been occasional debate regarding the use and merits of a multi-mission facility such as the FDF for ground navigation services versus the integration of all ground navigation functions in MOCs. We already have a history of moving the ground attitude determination functions from the FDF to MOCs. When computing systems were mainframes and funding was provided by a central organization, the use of multi-mission 
support facilities made sense from both a business perspective and technical perspective. With the introduction of distributed computing capability, the availability of COTS for flight dynamics functions and the increasing sustaining costs of a multi-mission facility, the use of a multi-mission support facility may not be the most cost effective. However, if the sustaining costs can be scaled to the needs of the users, there are advantages to having a facility that supports multiple users. For missions that use GPS for navigation but need ground based navigation services for the first few days of the mission, it is not cost effective to set up a system in the MOC to perform that short term support. The FDF can provide these services in a cost effective manner. Another advantage to a multimission support facility is that it helps maintain important navigation skills; support personnel gain experience in different types and conditions of missions. Knowledge of what to do in a contingency and having support personnel readily available and experienced in navigation can be an asset in case a mission has a contingency.

There is no single solution that fits all mission types and mission requirements. FDF personnel have often assisted in development and/or testing of navigation systems located in MOCs and the FDF has served as a backup during early mission operations for this type of approach. For the foreseeable future, operation of a multi-mission navigation facility (the FDF) continues to serve a major purpose. To summarize, we see the following advantages to using a multi-mission facility for meeting many (but not necessarily all) of GSFC's future navigation needs:

- The FDF provides a focus and a home for institutional expertise in operational orbit determination and tracking systems that can be made readily available for resolving spacecraft anomalies, providing consultation on all navigation issues and mission concept development.

- The cost of maintenance of navigation systems (including license fees for COTS software) can be shared by many customers.

- Certain FDF functions are clearly not easily located in MOCs and are multi-mission in nature. This includes tracking data evaluation.

- A larger and greater suite of navigation tools and supporting software systems are generally available for on-orbit analysis and troubleshooting in a multi-mission facility.

- All necessary interfaces with the many tracking networks are already available and tested with the FDF and do not have to be replicated for each new mission.

- FDF products and services remain cost competitive with other approaches for ground navigation support

Intuitively, these advantages go away as the mission set supported by the FDF becomes very small. The size of the FDF has been reduced and it is acknowledged that it must be scalable in the future to the mission set. This is one motivation for the reengineering effort discussed later in this paper. However, at some point the cost of services from a multi-mission facility will not be competitive due to certain fixed costs inherent with operating such a facility. Through the various FDF planning exercises, this crossover point has not been determined. In a qualitative sense, the feeling is that with the additional scalability that will be realized by continued reengineering of the facility, the FDF will remain viable as a multi-mission facility to the missions planned through the next decade.

\section{Current Drivers for Change}

While the present systems and software within the FDF are sufficient to support the current missions and could be modified to support new mission requirements, without a fundamental change in the architecture and approach to mission support, the FDF would be unable to take advantage of new or recent technology or respond to new mission requirements and implement system changes in a timely and cost-effective manner. The costs of maintaining legacy software and hardware increase as time goes by, which in turn increases the facility sustaining costs. Additionally, the ability of these legacy systems to support future missions decreases over time as missions become more complex or requirements change. Having a flexible and adaptable infrastructure assures that the FDF is able to support the present missions, and is able to adapt to future mission requirements with minimal impact.

Some of the drivers for facility modernization are mission requirements, cost reduction, and technology. Details of these drivers are given below. While these are not all inclusive, they represent the ones that we consider the major drivers for change in the FDF.

\section{Mission Requirements}

One of the biggest challenges for future FDF operations is to ensure the FDF staffing (both direct support staff and sustaining engineering support staff) is scaleable to the overall work flow so that the loss of one significant customer does not present increases in cost to other customers. An FDF "mission model" is maintained to provide insight into user needs and the projected level of activity in the FDF. Looking to the future, fewer missions will require services and the level of service will also change. In general, the need for full 
service flight dynamics support will decrease. However, the missions that do require FDF support throughout their lifetime tend to be more demanding and complex. Many near Earth missions are using onboard navigation and as a result only need support the first week after launch. Many robotic missions do not have 24/7 support and as a result, require some level of automation. Smaller and less-costly missions only require support for short periods or as backup/contingency.

Figure 3 depicts the latest mission model for the FDF. One uncertainty with the mission model is the role of the FDF with NASA's Constellation Program. FDF support to the STS accounts for approximately 20 percent of the work in the FDF (as measured by funding). Early discussions with JSC regarding support to the Orion manned vehicle have focused on FDF support similar to STS (backup orbit determination and generation of acquisition data). Whether or not the FDF ultimately has a role with the Constellation Program, there will be some loss of work after the retirement of the STS.

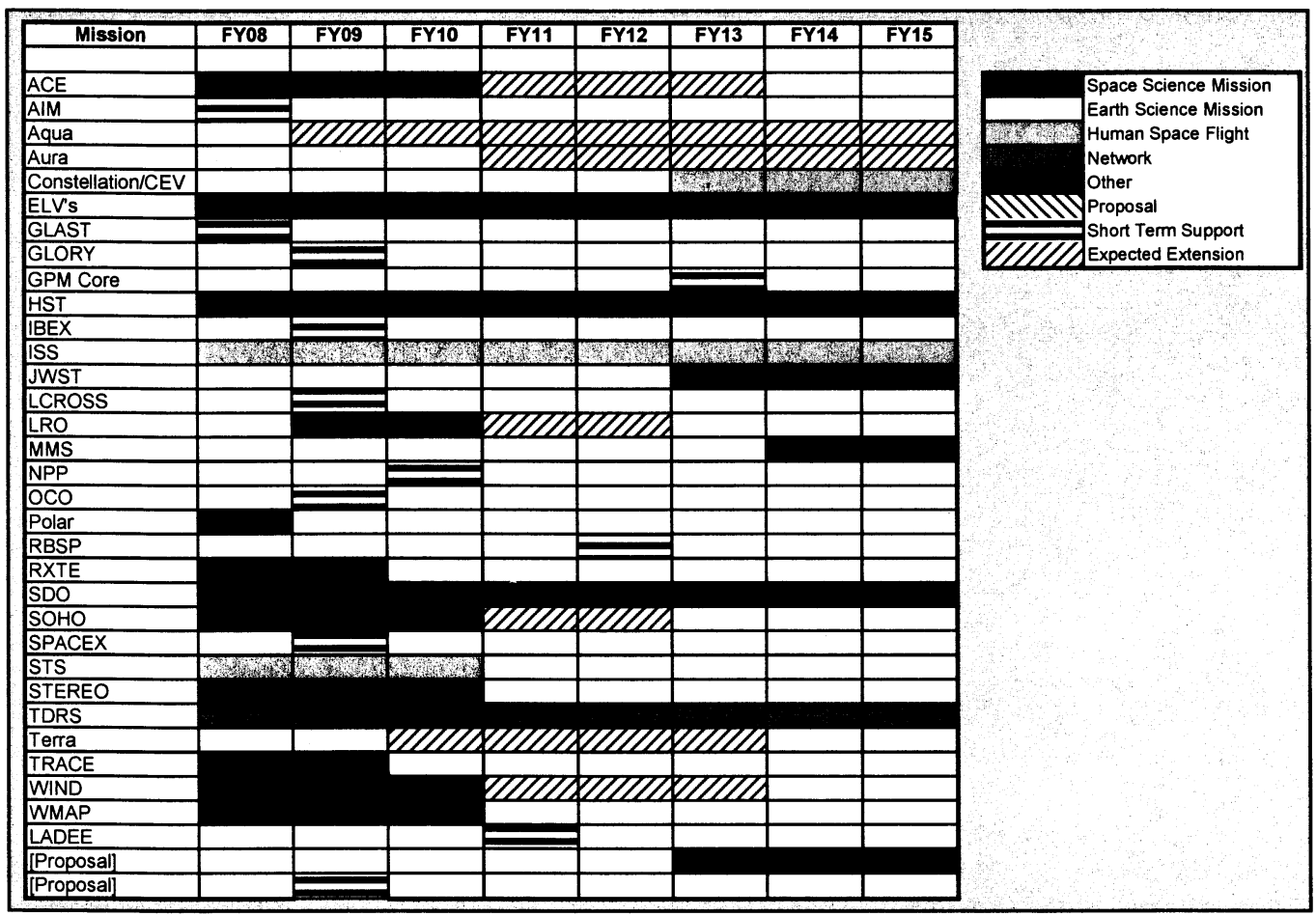

Figure 3. FDF Mission Model

\section{Cost}

Limited budgets for existing missions and a no-growth mission base do not allow for the upkeep of a large infrastructure. To maintain a viable facility we need to keep a customer base by supplying quality support for a reasonable and fair cost. This becomes a challenge without efficient and flexible systems and as more missions require only short-term support. FDF users pay for the "direct" cost associated with generating an FDF product. In addition, a key component of the cost passed to FDF users is the cost for sustaining engineering support and basic facility maintenance. This is the area with the greatest potential for cost reduction and is highly dependent on the FDF architecture.

\section{Technology}

There are many technologies that have been developed over the past ten years that are driving the future of the FDF. The use of the internet has changed the data communication for mission support. Most missions today use Internet Protocol (IP) and communicate over various networks. This has created the need to establish communications with multiple networks for operations. Network security is a major consideration. The use of databases allows for easier storage and retrieval of data. The computing hardware available today provides for more computational capability at a lower cost. The availability of COTS software reduces the need for legacy or 
in-house developed software; however the system must be more easily integrated into the FDF. Taking advantage of all of these requires a system that is easily adjusted and modified.

The concept of data-driven systems is being used in mission operations. Processes are executed based on the availability of data and delivery occurs when products are available. System automation and an architecture that supports lights-out operations are essential to providing cost-effective flight dynamics support.

\section{Fourth Generation FDF (2012): GMSEC-Based Architecture}

By 2004, it became obvious that the FDF required substantial upgrades to meet future mission requirements in a cost-effective manner. More important, the existing architecture was very inflexible with regard to integrating new applications packages, including commercial software systems for spacecraft navigation computations. While the facility continued to evolve in what can be characterized as incremental steps driven by near-term needs, the overall architecture and infrastructure of the FDF was in need of a substantial technical refresh given that much of the computer hardware, software, architecture and configurations were virtually unchanged since migration of the FDF off of mainframe computers in the mid-1990s. Unfortunately, establishing new business processes (in which we had to transition to a fully reimbursable operations facility) and the need for many short-term security upgrades took priority. An internal document ${ }^{5}$ produced in May of 2004 identified many of these short-term priorities. In early 2007 , an architecture study team was commissioned to analyze architecture options for the future. This team completed their efforts in late 2007 with a set of recommendations for FDF modernization.

\section{A. Architecture Study Recommendations}

The Architecture Study Team identified two overarching requirements for modernizing the FDF. First, the FDF architecture must support the mission model. The effect of this requirement is not only to scale the size of the facility and its architecture to the number of missions to be supported, but to also drive the need for greater flexibility to support project needs. For example, the JWST mission will have position determination accuracy requirements that will drive the need to use an orbit determination system different from the standard Goddard Trajectory Determination System (GTDS) used by most missions supported in the FDF. A COTS system is currently under evaluation for use by JWST. The architecture must be able to accommodate the rapid and low-cost integration of this and other new applications systems into the FDF.

The second overarching requirement is that the new architecture must reduce sustaining engineering and facility operations costs. These "indirect" costs are paid by FDF customers. While sustaining engineering and facility costs are now less than 30 percent of the same costs in 1992, they remain a significant cost incurred by FDF customers.

Consistent with the overarching requirements, the team performed analysis of architecture requirements and concluded that the new architecture must provide the following:

- Rapid and simple application system changes/upgrades

- Database data synchronization and automated failover

- Easy deployment of new communication interfaces with required security measures

- System-wide monitoring - fault detection, prevention, notification, automated recovery

- Receive / transmit data over multiple networks

- Publish / subscribe compliant applications that support interactive or automated modes

- Automatic product generation and delivery for routine operations

- Automation - routine for lights out; automated load distribution

- Security - support for NASA security standards

The architecture team did not perform an in-depth evaluation of all FDF applications software. However, the team did recommend that some legacy software systems be replaced or reengineered. In response to the architecture requirements, the final recommendation was to re-engineer the FDF with the Goddard Mission Services Evolution Center (GMSEC) Architecture. GMSEC is a data/event driven architecture that uses a publish/subscribe message bus middleware. Figure 4 depicts the FDF under the proposed GMSEC architecture. In addition to simplifying interfaces, the GMSEC architecture provides "situational awareness," system-wide control, and event driven automation. It also enables rapid introduction of new applications software and simplifies the addition of new tracking data formats and types. Adding credibility to the recommendation was an analysis of the "business case" for using the GMSEC architecture. This included an evaluation of operations savings (presented later in this paper). As a self-sustaining operation, reengineering resources must be paid by current FDF users. Thus, a major development activity with additional costs levied on FDF users was not a viable option. 


\section{B. GMSEC Fundamentals}

The GMSEC ground architecture development was begun in 2001. Today, it is a mature architecture that has been adopted by many of GSFC's flight project ground systems. ${ }^{6,7}$ It was developed out of the need to reduce operations costs, make it easier to incorporate new technology and COTS into ground systems, and reduce duplication of efforts between various ground system development efforts. The technical approach for GMSEC is based on several important concepts:

- Standardized interfaces - by standardizing interfaces and not components, the user is able to select the tool that best fits mission requirements.

- Middleware Infrastructure - GMSEC revolves around a message oriented middleware. This middleware can be commercial, open source or GSFC-developed.

- User chooses components - GMSEC allows the users to select the components that best fit their needs. The architecture also facilitates the use of legacy components.

- General purpose approach - GMSEC is designed to be usable for many different missions and domains. GMSEC was originally applied to spacecraft ground systems, yet it is well suited for the FDF system.

The GMSEC architecture uses a message bus for communication between processes or nodes. What used to be achieved with socket communications is now accomplished over the message bus. The processes or nodes only interface with the bus which simplifies communications for each component.

The publish/subscribe concept is used for passing information across the bus. An application will publish information (data) to the bus via messages. These messages have a subject name and message content. An application that needs this data or information will then subscribe to this message. The bus then delivers the message to the applications that subscribe to it.

Many middleware packages use the publisher/subscribe method; however, the message structure for a given middleware is proprietary. As a result, different types of middleware are not compatible with each other and an application written to interface with one middleware is not compatible with another middleware. GMSEC has addressed this issue by having a GMSEC Application Programming Interface (API). The API standardizes the capabilities of different middleware so that they appear the same to an application. Having a GMSEC API allows for change-out of middleware should a vendor go out of business, to switch to a lower cost middleware product or upgrade to a newer product. This GMSEC API supports many operating systems, languages and platforms. This makes it an important component in the GMSEC architecture.

In addition to the GMSEC API, GMSEC has message standards that facilitate the use of various applications within the architecture. These message standards make it easy for vendors and developers to make an application GMSEC-compatible and simplify the integration of applications.

A component is considered GMSEC-compliant if it meets certain standards. A component must 1) follow GMSEC standard message definitions; 2) publish a heartbeat message on a regular basis; 3) publish status/log messages and 4) be able to receive user directives for its control over the bus.

\section{Justification for GMSEC in the FDF}

Due to the extensive work at Goddard in developing the GMSEC architecture including the middleware and various support tools, it was not difficult to conclude the GMSEC Architecture will allow the FDF to upgrade and modernize with a modest investment. However, the most significant justification for adopting the GMSEC architecture is its ability to meet FDF modernization requirements for flexibility. The Architecture Study Team also offered the following rationale for moving ahead with a GMSEC based architecture:

- There is substantial corporate knowledge, compliant components, tools and support available for the GMSEC based architecture.

- GMSEC architecture is mature enough to allow immediate implementation.

- GMSEC architecture supports phased and gradual introduction.

- Use of the GMSEC architecture leverages prior (and substantial) investment in both time and money. Examples include:

- Evaluating and selecting communication mechanisms

- Developing message standards, interfaces, tools and mechanisms for communication

- Developing and validating tools for autonomy, failover and system monitoring

- Creating an extensive development environment with support tools and message generation and monitoring tools

- Successful history of multiple mission verification and validation

12

American Institute of Aeronautics and Astronautics 
- $\quad$ Supporting multiple platforms, languages and middleware

- $\quad$ Getting buy-in from the missions and establishing the new interfaces

- New mission data types and applications software can be developed and tested without interference with present operations.

- $\quad$ Less time spent testing and integrating into operations systems

- Overall development to operations time reduced

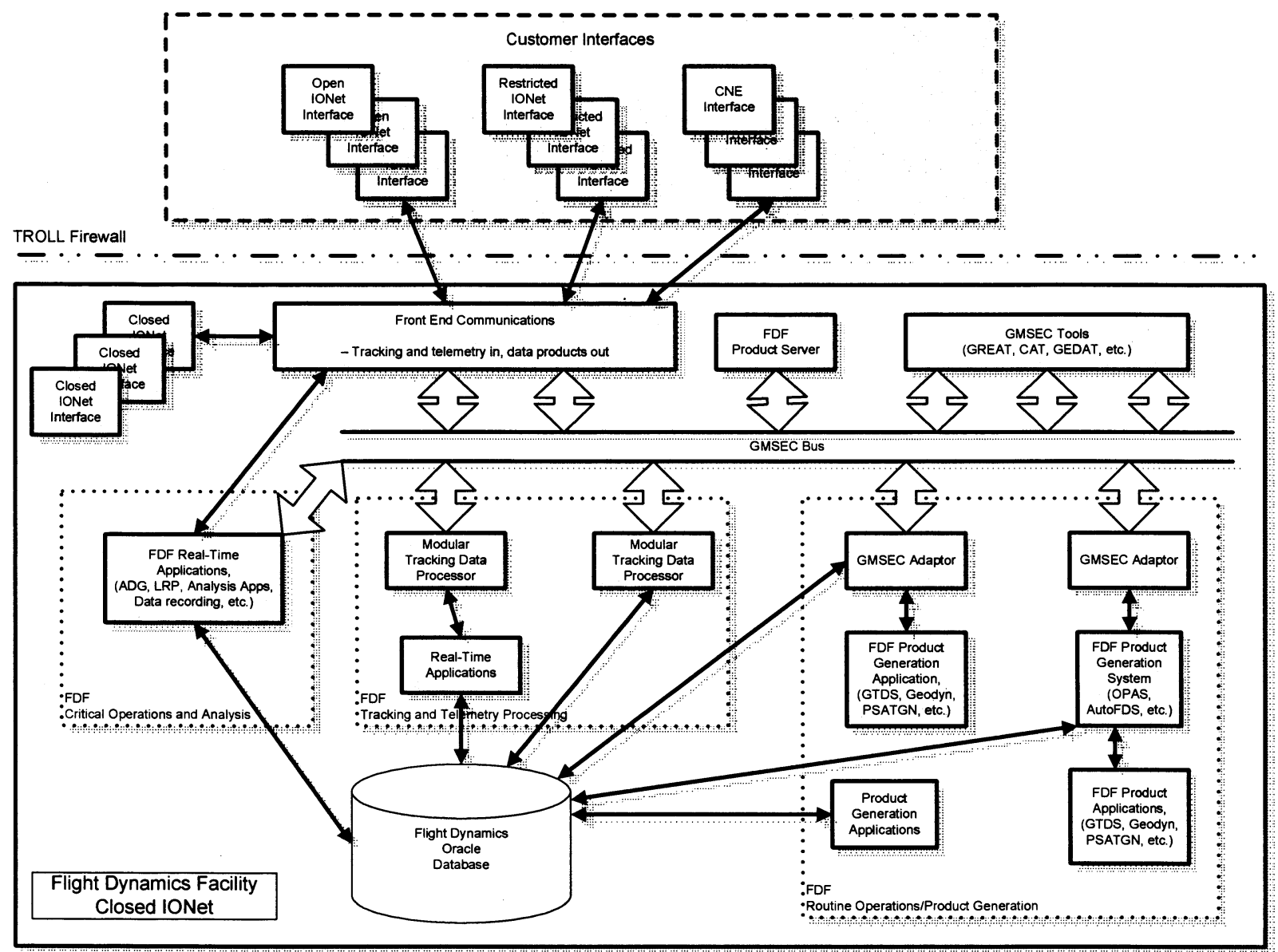

Figure 4. Future GMSEC-based FDF

Evolving to a GMSEC architecture will result in mission cost savings by reducing sustaining engineering staff. It will also enable new operations and introduction of new applications and tools. For example, MOCs will be able to connect directly to the FDF using secure GMSEC middleware connections and autonomously request and receive FDF products. In this model, the MOC would request services and data through GMSEC messaging, and the appropriate application would respond. The potential exists and is being explored for this service request model to be used between the FDF and other NASA centers.

\section{Return on Investment}

An initial estimate of the cost to implement the GMSEC architecture in the FDF was 40 staff-years, dependent on a number of factors including skill level of the development staff (prior experience with GMSEC is highly beneficial) and implementation period. A significant effort was expended to show how this level of cost could be justified based on cost benefits with the proposed GMSEC architecture. As previously stated, the GMSEC 
architecture supports phased and gradual introduction into an existing operations environment. This provides great flexibility in implementing the new architecture. This is especially important in an operation that is severely costconstrained and must make use largely (if not completely) of existing sustaining engineering staff for implementing the new architecture. Intuitively, GMSEC offers the FDF the following cost benefits:

- Improved efficiencies in operations

- Lower sustaining engineering costs

- Savings in implementing new capabilities

The study team did not quantify the cost benefits due to increased automation of operations processes. There have been significant gains in efficiencies in the past with automation ${ }^{2}$ and it was believed the GMSEC architecture would enable more automation of routine processes for orbit determination and product generation. However, the more significant cost benefits were determined to be found in lower sustaining engineering costs for the facility. This includes routine software maintenance activities as well as costs for "facility managers" to operate, monitor and troubleshoot system-wide operation of the FDF system. The first step in the analysis performed by the architecture team was to identify all elements of sustaining engineering costs (staff) and identify those that were fixed (security, property management, etc.) versus those that were architecture dependent (such as software maintenance). The architecture team worked with current sustaining engineering staff to determine the reduction in the cost of these "recurrent" architecture-dependent activities that could be realized with a reengineered FDF.

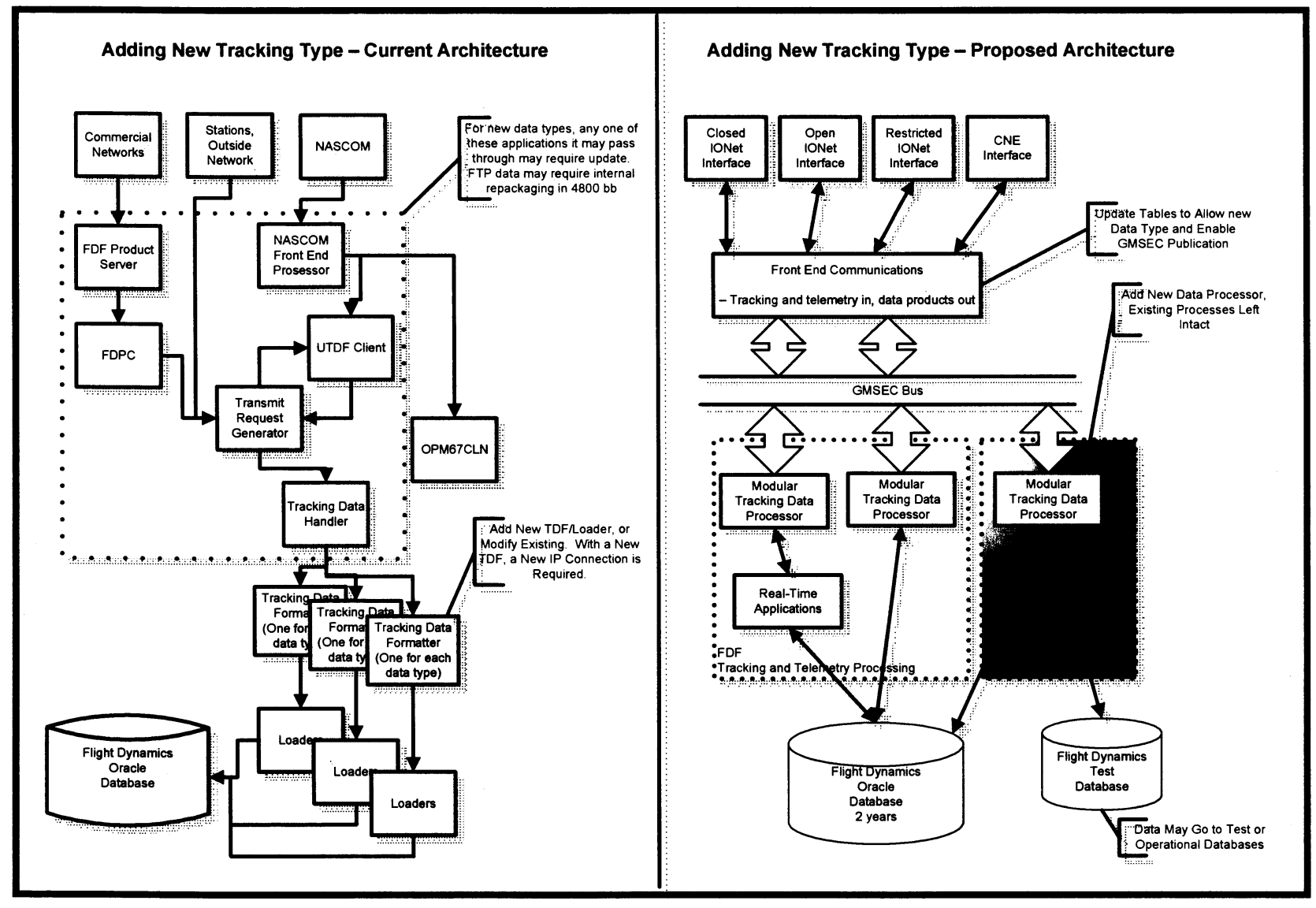

Figure 5. Tracking Data Modifications under Present and GMSEC Architecture 
More quantifiable savings were identified in the area of implementation of new FDF capabilities. The FDF must often add new tracking data types or new applications software to meet project requirements. A recent example is the addition of the new Track 2-34 tracking data format to the FDF orbit determination process. The study team examined this modification in detail to compare and contrast the process and costs for the modification under the current and the GMSEC architectures. These results are summarized in Table 2. Under the current architecture, these modifications were performed and required multiple changes to operations software systems. Under the GMSEC architecture, this new tracking data type would require modification to a single applications system and table updates (see Figure 5). An additional benefit is that development and testing can be performed without impact to any operations.

While GTDS will remain the primary orbit determination system within the FDF for some time to come, the FDF uses one COTS package for performing orbit determination for some of the missions it supports and will likely add other navigation packages in the future. Newer applications software systems for performing other FDF functions are also anticipated. These will be both in-house and commercial products. The study team also examined the cost of adding a new orbit determination application under the old and new GMSEC architectures and this is also summarized in Table 2.

Table 2. Estimated Savings with GMSEC Architecture

\begin{tabular}{|c|c|}
\hline \multicolumn{1}{|c|}{ Function } & $\begin{array}{c}\text { Estimated Savings with } \\
\text { GMSEC architecture } \\
\text { (staff years) }\end{array}$ \\
\hline $\begin{array}{c}\text { Sustaining Engineering } \\
\text { - Operations Engineer }\end{array}$ & $\begin{array}{c}2.0 / \text { year } \\
0.2 / \text { year }\end{array}$ \\
Hardware & Maintenance \\
System Engineering & Software \\
$\quad$ Maintenance & $0.2 /$ year \\
$\bullet \quad$ Total & $4.0 /$ year \\
\hline Addition of new tracking & \\
data format ( based on Track & $6.4 /$ year \\
2-34 modification) & 0.9 \\
\hline Addition of new Orbit & \\
Determination Application & $3.0-5.0$ \\
\hline
\end{tabular}

\section{E. Implementation Plan}

The recommendations of the Architecture Study Group were accepted by FDF management and endorsed by the Flight Dynamics User's Council earlier this year (2008). A plan to move forward with the implementation of the new architecture was also developed. This plan included the implementation phases, the Work Breakdown Structure (WBS), schedule and staffing. As a fully self-sustaining operation, the challenge of a major upgrade such as this is to find the resources (funding) to implement the new architecture. As stated previously, an effort of approximately 40 staff years was estimated. A five year implementation plan was initially proposed as a "reasonable" period to have most of the GMSEC architecture in place prior to support of the JWST mission and potential support to the NASA Constellation Program. This time period also appeared to work well with assumed resource limitations. With this schedule, more than 70 percent of the resource requirements can be met by reprioritizing current FDF system and software maintenance efforts, suspending any further maintenance on many legacy FDF software systems, and redirecting existing sustaining engineering staff. Thus, the challenge remains to find additional funding (approximately 12 staff years) for this effort. Various options are currently being explored by GSFC management. As Table 2 indicates, this investment would be paid back in FDF sustaining engineering cost savings in approximately 2 years. At the direction of GSFC management, the architecture team also developed a plan along with funding estimates for a three year implementation. As expected, this faster plan has a significant impact on required additional staffing. 
Implementation of the GMSEC architecture has begun and consists of three phases of development. Phase 1 will address the communications and data base processing infrastructure. It was determined that this was the area where the most improvement could be made with early benefits available to current missions as well as future missions. Phase 2 will focus on process engineering and upgrades of legacy applications. Legacy systems such as GTDS will be reengineered to interface with the GMSEC middleware. Recent reengineering of the Acquisition Data Generator (ADG) software has given the team confidence in its estimates of effort to do this and ability to meet schedules. Several legacy systems that perform front end processing of tracking data will be retired and a new front end communications processor will be brought on-line. Finally, phase 3 will address data delivery functions, incorporate new end-to-end automation for the generation and delivery of many FDF products, and complete reengineering of legacy applications. The reengineering effort is scheduled for completion by the end of 2012 .

\section{Summary and Conclusions}

The FDF is a multi-mission support facility that has been in existence for more than thirty years. It has evolved to meet continuously changing needs of NASA flight projects. A major upgrade to the FDF architecture is now necessary to both reduce sustaining engineering costs that are currently borne entirely by FDF users and to be able to meet requirements for rapid introduction of new flight dynamics applications and operations concepts for future missions. Adopting the GMSEC architecture should enable the FDF to continue to provide navigation services to missions in the future, and it has been shown to be cost-effective to implement this upgrade under the FDF's new business model.

This paper included a look back at some past planning efforts for the FDF and presented "lessons learned" for similar efforts in the future. With the completion of the recent architecture study and startup of the FDF reengineering effort, some additional comments and conclusions can be made:

1. The work of the architecture study has reinforced the belief that the FDF, as a multi-mission facility, remains a viable and cost effective source of navigation services. This paper was not intended to present a comprehensive defense for continued operation of a multi-mission operations facility for future mission navigation services. However, a reengineered FDF will continue to provide services that are sufficiently cost-competitive to maintain an influx of new business. There are other arguments that can be made for a centralized navigation facility (maintenance of core competency, security issues, etc.), but these need not be made if the cost of services is attractive to flight project customers.

2. Our experience seems to indicate that a strategic planning activity for a multi-mission facility such as the FDF must be performed at a minimum of every five years. The FDF has never maintained a formal strategic planning cycle. Our attempts at long-range planning have been spaced at roughly five-year intervals, usually by accident rather than a clear process that requires a periodic planning activity. While we may have benefited from a more vigorous process that emphasized more frequent updates to our long-range plans, the 5 year cycle has been adequate.

3. Maintaining a realistic mission model is essential. Many of the flaws in planning activities in the 1990's can be traced to not having a well understood mission model. Because it is mission-funded, the FDF must be more responsive and adaptable to changes in mission requirements (for example, short term early mission support to missions with autonomous navigation systems), and its size must be scalable such that individual users will not face cost increases for services as the FDF customer base changes.

\section{Appendix: Acronyms}

$\begin{array}{ll}\text { ADG } & \text { Acquisition Data Generator } \\ \text { API } & \text { Application Programming Interface } \\ \text { COMPASS } & \text { Combined Operational Mission Planning and Attitude Support System } \\ \text { COTS } & \text { Commercial Off-the-Shelf } \\ \text { DSN } & \text { Deep Space Network } \\ \text { ELV } & \text { Expendable Launch Vehicle } \\ \text { FDD } & \text { Flight Dynamics Division } \\ \text { FDDI } & \text { Fiber Distribution Data Interface } \\ \text { FDF } & \text { Flight Dynamics Facility } \\ \text { GLAST } & \text { Gamma-ray Large Area Space Telescope } \\ \text { GMSEC } & \text { Goddard Mission Services Evolution Center }\end{array}$




$\begin{array}{ll}\text { GN } & \text { Ground Network } \\ \text { GOSIP } & \text { Government Open Systems Interconnection Profile } \\ \text { GPS } & \text { Global Positioning System } \\ \text { GSFC } & \text { Goddard Space Flight Center } \\ \text { GTDS } & \text { Goddard Trajectory Determination System } \\ \text { HST } & \text { Hubble Space Telescope } \\ \text { IONet } & \text { Internet Protocol Operational Network } \\ \text { IP } & \text { Internet Protocol } \\ \text { ISS } & \text { International Space Station } \\ \text { JSC } & \text { Johnson Space Center } \\ \text { JWST } & \text { James Webb Space Telescope } \\ \text { LRO } & \text { Lunar Reconnaissance Orbiter } \\ \text { MIPS } & \text { Million Instructions Per Second } \\ \text { MMS } & \text { Magnetospheric Multiscale Mission } \\ \text { MOC } & \text { Mission Operations Center } \\ \text { MSFN } & \text { Manned Space Flight Network } \\ \text { NASA } & \text { National Aeronautics and Space Administration } \\ \text { NOAA } & \text { National Oceanic \& Atmospheric Administration } \\ \text { OPAS } & \text { Orbit Production Automation System } \\ \text { POSIX } & \text { Portable operating System Interface for Computer } \\ \text { SDO } & \text { Solar Dynamics Observatory } \\ \text { SN } & \text { Space Network } \\ \text { STEREO } & \text { Solar TErrestrial RElations Observatory } \\ \text { STS } & \text { Space Transportation System } \\ \text { TDRSS } & \text { Tracking Data Relay Satellite System } \\ \text { USN } & \text { Universal Space Network } \\ \text { WSC } & \text { White Sands Complex } \\ & \end{array}$

\section{Acknowledgments}

The authors would like to acknowledge the work of the FDF Architecture Study Team led by Rodger Abel. Members of the team included John Bristow, Joseph Sparmo, Patricia Johnson and Nancy Smith of Goddard Space Flight Center. Participants from the FDF software engineering team included Keith Chapman, Steve Gardner, and Steve Brower of aisolutions, Inc.; David Shefsky of SAIC; and Darla German and Robert Titus of Honeywell.

\section{References}

\footnotetext{
1"Flight Dynamics Division (FDD) 1990 Long-Range Plan," 550-FDD-91/001, January 1991

${ }^{2}$ Peters, K., "Flight Dynamics Division (FDD) Flight Dynamics Facility (FDF) System Architecture Study," 551-FDD91/016R0UD0, January 1992

${ }^{3}$ Chapman, K., C. Cox, C. Thomas, O. Cuevas, R. Beckman, "Production and Quality Assurance Automation in the Goddard Space Flight Center Flight Dynamics Facility," SPACEOPS 1994 Third International Symposium on Space Mission Operations and Ground Data Systems. 1995

${ }^{4}$ Stengle, T., S. Greatorex, K. Hartman, J. Jackson., "Flight Dynamics Operations Support Plan 2000,” unpublished, February

${ }^{5}$ Hoge, S.,J. Lynch, T. Stengle., "Flight Dynamics Vision 2005," unpublished, October 2003

${ }^{6}$ Smith, D., J. Bristow, P. Crouse, "Reducing Development and Operations Cost Using NASA's "GMSEC" Systems Architecture," $7^{\text {th }}$ International Symposium on Reducing the Cost of Spacecraft Ground Systems and Operations," Moscow, June 2007

${ }^{7}$ Surka, D., L. Jones, P. Crouse, E. Cary, T. Esposito, “Integrating Automation into a Multi-Mission Operations Center," AIAA

${ }^{8}$ Hoge, S.., "State of the Flight Dynamics Facility: An Assessment of the FDF 90 Days After the Start of the MOMS Contract," unpublished, May 2004
} 